An online, single-session intervention for adolescent self-injurious thoughts and behaviors:

Results from a randomized trial

Mallory L. Dobias, M.A. ${ }^{* a}$ (ORCID: 0000-0001-6814-3006)

Jessica L. Schleider, Ph.D. a (ORCID: 0000-0003-2426-1953)

Laura Jans, B.S. ${ }^{\text {a }}$

Kathryn R. Fox, Ph.D. ${ }^{\text {b }}$ (ORCID: 0000-0002-2697-7771)

*Corresponding author: Mallory L. Dobias, M.A. (mallory.dobias@stonybrook.edu)

${ }^{a}$ Department of Psychology, Stony Brook University, Stony Brook, NY, USA,11794-2500

${ }^{\mathrm{b}}$ Department of Psychology, University of Denver, Denver, CO, USA, 80208

This manuscript is now published at Behaviour Research and Therapy (December, 2021).

Link to published article: $\underline{\text { https://doi.org/10.1016/j.brat.2021.103983 }}$

Citation: Dobias, M.L., Schleider, J.L., Jans, L., \& Fox, K.R. (2021). An online, single-session intervention for adolescent self-injurious thoughts and behaviors: Results from a randomized trial. Behaviour Research \& Therapy. doi: 10.1016/j.brat.2021.103983 


\begin{abstract}
Background: Across 50 years of research, existing interventions for self-injurious thoughts and behaviors (SITBs) in adolescents have remained largely ineffective and inaccessible. Singlesession interventions, interventions designed to last one session, may be a low-cost and timely resource for adolescents engaging in SITBs who may not otherwise receive treatment. Method: 565 adolescents $\left(M_{\mathrm{age}}=14.95\right.$ years $)$ endorsing recent engagement in non-suicidal self-injury (NSSI) were randomized to receive a 30-minute, web-based, single-session intervention"Project SAVE" — or an active, attention-matched control program. Proximal outcomes were measured at baseline and immediately post-intervention. Long-term outcomes were measured at baseline and 3-month follow-up. Results: Adolescents rated Project SAVE as acceptable; 80\% of participants randomized to SAVE completed the intervention. Between-group effects were non-significant for pre-registered outcomes: post-intervention likelihood of future NSSI; 3month frequencies of NSSI and suicidal ideation at follow-up. Relative to control-group participants, SAVE participants reported short-term improvements in two exploratory outcomes: self-hatred $(d=-.35, p<.001)$ and desire to stop future NSSI $(d=.25, p=.003)$. Conclusions: Project SAVE is an acceptable resource for adolescents engaging in SITBs-with short-term effects on clinically-relevant outcomes. Future research may evaluate SAVE as an easy-toaccess, short-term coping resource for youth engaging in SITBs.
\end{abstract}

ClinicalTrials.gov Identifier: NCT04498143

Keywords: Adolescents, Self-Injury, Suicide, Digital Mental Health, Intervention 


\section{An online, single-session intervention for adolescent self-injurious thoughts and behaviors: Results from a randomized trial}

Self-injurious thoughts and behaviors (SITBs) often first emerge in adolescence (Nock et al., 2008, 2013). A substantial portion of adolescents have experienced, or engaged in, SITBs at some point in their lifetime - ranging from $\sim 17 \%$ for non-suicidal self-injury (NSSI) like cutting or burning oneself without intent to die (Swannell et al., 2014) to 20-24\% for suicidal ideation (Nock et al., 2008). Considering NSSI and other SITBs cause considerable distress for many youth, and that these predict future suicidal behavior (Asarnow et al., 2011; Cox et al., 2012; Ribeiro et al., 2016; Whitlock et al., 2013), albeit weakly, there is considerable need for detection and intervention in this age group. However, despite $>5$ decades of research dedicated to the prevention and treatment of SITBs, rates of SITBs among adolescents have remained stable and high (Nock et al., 2008). Timely, youth-directed interventions for SITBs are critical. To help address this need, this nationwide randomized trial aimed to test whether a brief, online intervention designed to target SITBs could reduce short-term outcomes of direct clinical importance — intentions to stop self-harming and self-hatred—as well as past 3-month frequencies of non-suicidal self-injury and suicidal ideation in adolescents reporting recent SITBs.

The State of the Science: Limits of Existing Treatments. Years of SITB intervention research have consistently observed small, inconsistent effect sizes (Brausch \& Girresch, 2012; Glenn et al., 2015; Gonzales \& Bergstrom, 2013; Nock, 2010). While the number of randomized trials for SITB treatments have increased at a "near-exponential" rate since 1970, a 2020 metaanalysis indicates intervention effects have not improved over time (Fox, Huang, et al., 2020). Further, the limited accessibility of many existing SITB interventions might exacerbate the 
problem of low intervention efficacy. Many adolescents experiencing SITBs simply do not access any mental health-specific services. More than two thirds of adolescents who report pastyear suicidal ideation - and more than half of those with a suicide plan or attempt in the past year-report having no past-year contact with a mental health specialist (Husky et al., 2012).

High attrition and low uptake are persistent issues for the subset of adolescents who do seek help (Granboulan et al., 2001; Lizardi \& Stanley, 2010; Piacentini et al., 1995; Trautman et al., 1993). Among adolescents who recently attempted suicide, more than a quarter attended no scheduled outpatient sessions post-emergency department discharge, and more than one in 10 adolescents attended only a single session (Granboulan et al., 2001). For over a decade, SITB intervention research has outlined a need for briefer and more accessible mental health supports (Lizardi \& Stanley, 2010; McCabe et al., 2018; Stanley \& Brown, 2012; Stewart et al., 2019). However, clinical reality has yet to sufficiently address this need—with many offered treatment protocols requiring 6 or more months of in-person sessions to complete (Brent et al., 2009; Esposito-Smythers et al., 2011; Rossouw \& Fonagy, 2012; Taylor et al., 2011). Thus, brief, accessible mental health interventions for SITBs among adolescents could have a substantial positive impact for those who would not otherwise access treatment.

Single-Session Interventions: A Promising Path Forward. Developing well-targeted single-session interventions (SSIs) is one way to maximize treatment accessibility, acceptability, and potency (Schleider et al., 2020). Very brief treatments designed to last one session, SSIs directly address many traditional barriers to treatment access (time, transportation, money, etc.). Additionally, youth-initiated, web-based SSIs circumvent the "parent gatekeeper effect", where many adolescents go without mental health care in the absence of parental knowledge or support (Stiffman et al., 2004) — a problem particularly relevant for youth with SITBs who may not feel 
comfortable asking for support and who prefer their parents/guardians not know about their SITB engagement. Given their brief format, SSIs also encourage intentional decision-making around the selection of treatment content and structure. Despite a wide range of existing treatment mechanisms, no treatment for SITBs is especially efficacious (Fox, Huang, et al., 2020). Therefore, shortcomings of existing SITB treatments could well-inform the development of a SSI that utilizes a very different framework for SITB intervention: one that de-stigmatizes difficult thoughts and feelings (including self-hate) that a youth might be facing; works collaboratively, yet anonymously, with the youth to develop personalized coping strategies to maximize relevance and potency; empowers the youth to initiate their own path to recovery; and demonstrates the value of the youth's lived experience by asking them to share their coping strategies with others.

Indeed, single-session interventions — including those using this framework — have been shown to ameliorate adolescent psychopathology. In a 2017 meta-analysis of 50 RCTs $(10,508$ youth participants), SSIs successfully reduced diverse youth mental health difficulties (mean $g=$ .32), with no moderation effects of intervention delivery modality (e.g. therapist-delivered vs. web-based), youth problem severity (diagnosed disorder vs. subclinical symptoms), or treatment length (range: 5 minutes to several hours) on treatment effect size (Schleider \& Weisz, 2017). As one example, a single 30-minute, web-based SSI decreased depression in high-symptom adolescents 9-months later (parent-report: $d=.60$; youth-report: $d=.32$; Schleider $\&$ Weisz, 2018). More recently, a 2021 RCT found two online single-session interventions decreased depressive symptoms 3 months later, compared to an active control (Schleider et al., 2021). Although SITBs are often considered high-severity problems, existing literature suggests shorter treatments may also help reduce clinically severe difficulties. Two systematic reviews report no significant 
moderating effect of treatment length on suicidal ideation or non-suicidal self-injury (i.e. single vs. multiple sessions and number of weeks in treatment, respectively (Ougrin et al., 2015; Labelle et al., 2015). More recently, a comprehensive meta-analysis of 345 SITB treatments found no moderating effect of treatment length on SITB treatment outcomes (Fox, Huang, et al., 2020). Initial evidence supports the creation and evaluation of novel, accessible SSIs targeting modifiable factors underlying SITBs.

Considering Novel Targets for Brief SITB-Focused Interventions. Prior interventions have failed to sustainably reduce youth SITBs (Fox, Huang, et al., 2020). This lack of progress may, in part, be due to difficulty selecting appropriate treatment targets (Franklin et al., 2018). Many existing SITB interventions are designed to target theory-driven constructs that co-occur with — and predict future episodes of-SITBs. For example, research consistently identifies negative views about the self (e.g., self-hatred, self-criticism) as a correlate and risk factor for NSSI engagement across the lifespan (Fox et al., 2018; Pfeiffer et al., 2014; Smith et al., 2020; Turnell et al., 2019; Zelkowitz \& Cole, 2019). Further, research in samples without active SITBs indicates that negative self-beliefs are indeed modifiable when targeted via intervention (Halamová et al., 2018; Luoma \& Platt, 2015; Schleider et al., 2020). However, recent evidence suggests that modifying negative self-beliefs in the short-term may be insufficient, on its own, to produce long-term changes in self-directed violence in those with a longstanding history of such behaviors (Hooley et al., 2018). Broader calls to move away from choosing treatment targets based on SITB correlates and risk factors (Franklin et al., 2018) — which may or may not have causal relationships with SITBs - also suggest that negative self-beliefs (such as self-hate, on its own) may not be an ideal target for SITB intervention. 
Novel interventions may better reduce youth SITBs by aiming to reduce self-injurious behaviors that occur in the context of negative self-beliefs - rather than attempting to change SITBs indirectly via targeting self-criticism or self-hatred. Negative self-beliefs are highly prevalent and distressing among young people who engage in NSSI (Burke et al., 2021; LayeGindhu \& Schonert-Reichl, 2005), and desire for self-punishment is one commonly-endorsed reason that many adolescents engage in self-harming behavior (Doyle et al., 2017; Klonsky, 2007). Thus, focusing SITB interventions on one's capacity to respond flexibly in the face of negative self-beliefs_-for instance, by engaging in alternative coping strategies to self-harmmay therefore add value to SITB-focused interventions. Stated plainly, novel interventions might seek to empower youths to "decouple" the experience of negative self-beliefs (e.g., self-hatred) from resulting NSSI by providing psychoeducation and introducing alternative coping methods to replace self-harm. However, this prospect has not yet been evaluated formally via a SSI format.

The Present Study. This nationwide randomized trial tested the acceptability, short-term utility, and 3-month effects of an accessible online SSI, "Project SAVE" (Stopping Adolescent Violence Everywhere). Project SAVE was designed to target and reduce frequency of NSSI and suicide ideation in adolescents with a recent history of SITBs and negative self-beliefs via (1) normalizing and de-stigmatizing feelings of self-hatred, and (2) empowering youth to select alternative coping strategies in the face of self-hatred. Goals were three-fold. First, we evaluated the acceptability of the Project SAVE intervention as indexed by adolescents' quantitative feedback and intervention completion rates. Second, we tested whether Project SAVE improved pre-registered outcomes of interest - relative to the control. For our primary outcome, we evaluated 3-month frequency of NSSI in both groups 3 months post-intervention. As secondary 
outcomes, we assessed likelihood of future NSSI immediately following the intervention, and 3month frequency of suicidal ideation 3 months later. Third, we examined whether Project SAVE improved two exploratory outcomes - desire to stop non-suicidal self-injury and self-hatredrelative to the control. This trial represents the first large-scale RCT testing a one-session, selfguided intervention versus an active, attention-matched control for adolescents engaging in selfinjury. Results may inform future efforts to strengthen the acceptability and potency of existing supports for youth experiencing SITBs.

\section{Method}

All study procedures were approved by the University of Denver Institutional Review Board and were pre-registered on ClinicalTrials.gov (identifier: NCT04498143) and Open Science Framework (https://osf.io/x 5cd9) prior to the enrollment of the first study participant.

\section{Participants}

Considering that a majority of RCTs testing treatment effects on SITBs (78.89\%) has been conducted in samples with $<500$ participants (Fox, Huang, et al., 2020), we pre-registered a sample size of $N=500$ for the current study. All participants were recruited from within the United States via advertisements on online social networking sites. To be included in the final analysis, each participant must have met the following criteria: aged between 13 and 16 years; comfortable reading and writing in English; no self-reported learning disability, visual impairment, or other difficulty that would make it difficult to answer questions on a computer; had a laptop, computer, or smartphone with internet access; reported engaging in NSSI within the previous month; reported disliking or hating themselves; did not exit the study prior to randomization to a study condition. Further, to be included, participants met additional data quality criteria as indexed by their qualitative responses (i.e., did not respond with: direct 
copying/pasting survey text, lack of English fluency, random text, or three words or fewer when asked for two sentences or more; see study pre-registration for more information, https://osf.io/x 5cd9). Although not formally pre-registered as an exclusion criterion, the present analysis also excluded participants who self-disclosed that they had skipped through or not read entire portions of the survey or online program. The above criteria yielded a final sample of 565 participating adolescents.

\section{Measures}

Although the present study included a broad range of questionnaires to facilitate exploratory secondary analyses, we report the instruments used to determine participant eligibility and measures of the trial's primary and secondary outcomes of interest below. A full list of study measures is available in the clinical trial registration (NCT04498143).

\section{Screening Survey}

English Fluency and Internet Access. The initial screening survey contained several single-item eligibility questions to ask if participants could read and write fluently in English, could read and answer questions on a computer, and had access to a laptop, smartphone or computer with internet connection. Participants who answered "no" to any of these questions were not eligible to participate in the present study.

Negative Views About the Self. A single item from the Children's Depression Inventory-2 (CDI-2; Kovacs, 2015), a 27-item scale assessing youth depression symptoms, was used to screen for negative views of the self. For each item, youth select one of three statements that best represents how they have been feeling within the past two weeks. Here, item 7 of the CDI-2 was used to select participants for inclusion who reported disliking or hating themselves 
(i.e. "I do not like myself" or "I hate myself”). Participants who answered, "I like myself”, in response to CDI-2 item 7 were ineligible for participation in the current study.

Past-Month Self-Injurious Behavior. The screening survey contained two, single-item questions adapted from the Self-Injurious Thoughts and Behaviors Interview-Revised (SITBI-R; Fox, Harris, et al., 2020): "Have you ever purposely hurt yourself without wanting to die (e.g., cutting, burning, hitting self, scratching, skin damage) causing skin damage (e.g., bruises or blood)?" and, "Have you purposefully hurt yourself without wanting to die (e.g. cutting, burning) within the past month?". Individuals who responded "no" to either of these two items were ineligible for participation.

\section{Baseline, Post-Intervention, and Follow-Up Questionnaires}

Demographics. After starting the baseline survey, participants answered a series of questions about their demographic information — including age, race/ethnicity, and gender identity.

Self-Injurious Thoughts and Behaviors. Items from the SITBI-R (Fox, Harris, et al., 2020) were used to measure both proximal and long-term SITB frequency outcomes of interest. Proximal outcomes (self-reported likelihood of future NSSI, desire for future NSSI) were measured at baseline and immediately post-intervention (SAVE or active control program). For likelihood of future NSSI (pre-registered, secondary outcome), participants answered a single question ("what do you think the likelihood is that you will purposefully hurt yourself without wanting to die in the future?") on a 5-point Likert scale: 0 (“"not at all”), 1 (“a little bit”), 2 ("somewhat"), 3 ("very much"), or 4 ("extremely"). To measure desire to stop future NSSI (exploratory outcome), participants were asked, "how much do you want to stop purposefully hurting yourself without wanting to die?", and told to rate their answer: 0 ("I don't want to stop 
at all”), 1 ("I want to stop a little bit"), 2 ("I want to stop somewhat"), 3 ("I want to stop very much"), or 4 ("I definitely want to stop").

For longer-term SITB outcomes (past 3-month frequencies of NSSI and SI), items from the SITBI-R questionnaire were administered at baseline and again at follow-up 3 months later. Participants were asked to report the number of times they had purposefully hurt themselves without wanting to die (pre-registered, primary outcome), and on how many days they had had thoughts of suicide for more than a few minutes (pre-registered, secondary outcome), within the past three months.

Self-Hatred. Self-hatred (exploratory outcome) was measured at baseline, immediately post-program, and again at 3-month follow-up using the Self-Hate Scale (SHS; (Turnell et al., 2019). The SHS is a reliable, valid 7-item questionnaire with excellent internal consistency within teenage samples (Schleider et al., 2020). The scale asks individuals to rate on a sliding scale the degree to which each statement (e.g. "I hate myself") is true for them, with anchors at 1 ("not at all true for me"), 4 ("somewhat true for me"), and 7 ("very true for me"). Here, the measure was adapted to instruct participants to rate each item based on how they were feeling "right now, in this moment".

Program Feedback. Acceptability of each intervention program was measured using the Program Feedback Scale (PFS; Schleider et al., 2019). The PFS is a 7-item questionnaire designed to evaluate participant feedback for web-based intervention programs. Individuals are asked to rate the extent to which they agree with each of the 7 statements (e.g. "the activity was easy to use") on a 5-point Likert scale: 1 ("really disagree"), 2 ("disagree"), 3 ("neutral"), 4 (“agree"), or 5 ("really agree"). In line with previous work testing online SSIs for youth mental health (Schleider et al., 2020), we pre-registered a mean score of $\geq 3.5$ as indicating acceptability 
of the online program in question. Additionally, means and standard deviations were calculated for each individual item.

\section{Online Programs}

\section{Project SAVE}

Project SAVE (“Stop Adolescent Violence Everywhere”) SSI (Dobias, Schleider, \& Fox, 2020; see https://osf.io/vguf4/) is a 30-minute, self-administered, web-based program that draws on components of cognitive behavior therapy (e.g. psychoeducation about relationships between thoughts, feelings, and behaviors; secondary coping skills such as distress tolerance) designed to decrease self-injurious behaviors in youth. The Project SAVE SSI has 4 general content sections: first, explaining the science behind how changing your actions (i.e., decreasing self-injurious behaviors) can positively impact your thoughts and emotions over time; second, detailing scientific evidence and testimonials from other teens that have decreased their selfinjurious behaviors and noticed positive change as a result; third, providing evidence-based tips and alternative coping mechanisms to replace self-harming behavior; and fourth, offering an opportunity for youth to share their own thoughts and advice on what they have learned with other teenagers who are facing similar challenges. These content areas are reinforced by intervention design features intended to maximize persuasiveness and memorability, in line with other efficacious, web-based SSIs for adolescents (Schleider et al., 2020). The majority of examples, adolescent testimonials, and content throughout the Project SAVE intervention discuss self-harm in the context of self-punishment and self-hatred. However, while indicating personal barriers to discontinuing self-harming behavior, adolescents had the option to select from the two most-commonly endorsed reasons for self-harm (Klonsky, 2007) they see as most closely aligning with their own reasons and barriers: self-punishment (i.e., "I feel like I deserve to feel 
badly"), emotion regulation ("nothing else helps me feel better in the moment"), or both.

Adolescents were then provided with additional psychoeducation and coping strategies specific to their barrier(s) of choice. As such, the Project SAVE intervention was designed to help adolescents "decouple" the relationship between their overwhelming or distressing emotional experiences — particularly self-hatred — and their engaging in self-harming behavior.

\section{Active Control Program}

Supportive Therapy (“Share Your Feelings”) SSI (Schleider \& Weisz, 2019; see https://osf.io/u4axs/) is a $~ 30$-minute, self-administered, web-based program that uses components of supportive therapy to encourage feelings sharing. Like Project SAVE, this SSI takes approximately 30 minutes to complete and is self-administered online. The supportive therapy SSI encourages participants in the control group to identify and express their feelings by: first, explaining why sharing feelings is natural, important, and helpful; second, including testimonials from teens who have shared their feelings with close others. In previous SSI trials, the supportive therapy SSI has had less favorable outcomes on depression in high-symptom adolescents when compared to an active treatment SSI (Schleider \& Weisz, 2018).

\section{Procedure}

Participant recruitment and baseline data collection were completed in August and September 2020. All participants were recruited from within the United States via advertisements posted on Instagram. Given that LGBTQ+ youth are at greater risk of NSSI and suicidal ideation relative to their cis-gender, heterosexual counterparts (Batejan et al., 2015; Fox, Choukas-Bradley, et al., 2020; Kidd et al., 2016; Marshal et al., 2011)—and many LGBTQ+ youth face additional barriers to mental health treatment access (e.g., fears of being "outed" or marginalized by mental health care providers (Brown et al., 2016) — many of the present study's 
advertisements were specifically designed to reach LGBTQ+ youth (e.g., deployed using LGBTQ-specific Instagram hashtags; included a pride flag graphic). After clicking on an advertisement for a research study (e.g., "Psychology Research Participants Wanted;" “Help researchers understand how teens think and feel;" "the study is completely confidential and done online”), participants were directed to a brief eligibility survey (6563 total responses collected). Of these responses, 863 individuals met initial inclusion criteria, completed the eligibility survey, submitted an online assent form, and provided a valid email address to receive information for the remainder of the study. Because the study posed minimal risk to participants, and because it was deemed logistically difficult if not impossible to secure parental consent online, parental consent was waived to decrease logistical barriers to participation and to increase willingness to participate in research among marginalized adolescents who might otherwise be underrepresented in mental health treatment research (e.g., LGBTQ+ youth who are not "out" to their parents; Smith et al., 2021). Participants who did not meet the present study's inclusion criteria were directed toward other, related opportunities to participate in mental health research and provided with a list of mental health resources.

Following the completion of this first survey, eligible individuals were asked to complete a second survey within 48-hours that included all baseline questionnaires, a 30-minute online program (either Project SAVE or the control program, per automated, 1:1 randomization in Qualtrics - a secure data collection software), and post-intervention questionnaires. As a result, both participants and study investigators were masked to participant condition throughout the entire data collection process. After excluding duplicates and participants who failed a second inclusion criteria check (aged between 13 and 16 years, endorsed NSSI within the past month, and did not exit the study prior to condition randomization), the study team collected baseline 
data from 578 eligible participants. Lastly, participants were asked to complete a final, follow-up survey containing questions about long-term outcomes approximately 3 months after completing the survey with their assigned program. Participants earned up to \$30 USD for their participation, via a 1-in-10 chance of winning $\$ 25$ after the baseline survey plus a guaranteed $\$ 5$ awarded upon follow-up survey completion. All follow-up data collection was completed November 2020 through January 2021.

The final group of participants included in present analyses $(N=565)$ was identified after reviewing participants' qualitative data and making final exclusion decisions based on preregistered data-quality criteria (copy/pasting responses from survey text; lack of English fluency; random text responses; responding with three words or fewer when writing prompts requested 2 sentences or more). Additionally, responses where participants explicitly acknowledged skipping or not reading portions of the survey were also excluded - resulting in five total data quality criteria. Participants were flagged for potential exclusion if their qualitative responses contained one or more of these five criteria.

To identify these participants, two independent coders were trained to flag responses based on the five criteria: first, two primary coders and one secondary coder independently coded responses from the first 50 participants before reviewing their responses as a group; second, the same two primary coders independently coded qualitative responses for a subset of participants $(\sim 20 \%)$ to establish reliability (i.e. kappa $>.60$ for all five coding categories); third, remaining responses were split between these two coders for review. The final list of "flagged participants" then brought to the full study team, where final exclusion decisions were made based on group consensus. This process yielded a final dataset containing 565 adolescents (see full CONSORT diagram in Figure 1 for details regarding exclusion decisions). 
[Figure 1: no color needed in printed figure]

Several procedures were in place to protect participants' safety throughout the course of their participation. All participants were provided with a list of electronic and LGBTQ+ friendly, online mental health resources. Any adolescent endorsing recent thoughts of suicide or past suicide attempts were directed toward a self-guided, online safety planning tool before the survey's conclusion (https://osf.io/rwktm/). Additionally, participants were explicitly asked whether they would like to talk with a member of the study team about their suicidal thoughts, or whether they would like to have a member of the study team contact their parent or guardian. A total of 135 participants indicated that they would be interested in a follow-up contact with study staff members after completing the baseline survey; however, only 21 responded when study staff reached out to these individuals to ask what additional support would be useful. Of those who responded, the most common request was for tips for talking to parents about mental health difficulties, and/or how to access mental health treatment without parent involvement. In other cases, participants asked for tips for coping or tips for how to manage difficult life circumstances. Given the very low number of participants who made additional contact with the study team, we did not control for these contacts in the present analyses (outlined below).

\section{Analytic Plan}

\section{Pre-Registered Analyses}

Intervention Acceptability. First, we evaluated acceptability of the Project SAVE intervention as indexed by adolescents' Program Feedback Scale scores — using a pre-registered mean score of $\geq 3.5$ to indicate acceptability. Additionally, we calculated the percentage of adolescents who completed the intervention out of the total number of individuals randomized to the Project SAVE condition. 
Intervention Effects on Study Outcomes. Second, we evaluated effects of the Project SAVE intervention on pre-registered outcomes of interest, relative to the control group. In accordance with our pre-registration, we checked for differential dropout by condition and imputed missing data prior to assessing intervention effects.

Differences In Dropout. Differential rates of dropout between study conditions can introduce bias in the interpretation of trial results. Therefore, we used Z-tests of differential proportions to evaluate whether the proportion of participants that "dropped out" before completing outcome measures significantly differed between intervention and control groups at post-intervention and follow-up. A p-value less than 0.05 would indicate significant differences in the proportion of dropout by condition.

Data Imputation. We imputed any missing data in our outcomes of interest. To do this, we executed an expectation-maximization and bootstrapping algorithm via the Amelia II package in $R$ (Honaker, King, \& Blackwell, 2011). For each outcome, we imputed a number of datasets corresponding to the percentage of missing data for that outcome, rounding to the next whole number. For example, an outcome with $19.2 \%$ missing data would require 20 imputed datasets. This approach allowed for rigorous intent-to-treat analyses that preserved power in the context of missing data.

Effects on Primary Outcome. After checking for differential dropout and imputing missing data, we tested intervention effects on our primary outcome of interest: frequency of NSSI within the past 3 months, measured at 3-month follow-up. For this outcome, we winsorized outliers - defined as values greater than the 95th percentile value - using the Winsorize function within the DescTools package in $R$ (Signorell et al., 2020). Next, we used Akaike Information Criterion (AIC) and Bayesian Information Criterion (BIC) values to select the most appropriate 
of four possible regression approaches (Poisson regression, negative binomial regression, zeroinflated Poisson regression, or zero-inflated negative binomial regression). We conducted the analysis associated with the lowest AIC and BIC values across all imputed datasets. Study condition and past 3-month frequency at baseline were included as predictors of the outcome.

Effects on Secondary Outcomes. We next tested whether a proximal outcome-selfreported likelihood of future NSSI - differed between intervention and control groups immediately following the completion of a program. We used condition assignment and selfreported likelihood of future NSSI at baseline to predict post-intervention likelihood of future NSSI in a multiple regression model. We checked all assumptions of multiple regression prior to interpreting model results (i.e., linear relationship between the independent variables and dependent variable; normally distributed errors; homoscedasticity; no perfect multicollinearity; influential observations).

As with our primary outcome (past 3-month frequency of NSSI), we winsorized outliers and calculated AIC and BIC values to select the most appropriate model choice for a secondary, long-term frequency outcome: past 3-month frequency of suicidal ideation. Using the selected regression type, we tested whether suicidal ideation frequency significantly differed between conditions at 3-month follow-up — controlling for suicidal ideation frequency at baseline.

Correcting for Multiple Tests. We used the false discovery rate (FDR; Benjamini \& Hochberg, 1995) method to correct for multiple tests, such that corrected p-values for study condition needed to equal $<.05$ for us to interpret a significant effect of condition on our outcome of interest. Between-group effect sizes (Cohen's $d, 95 \%$ confidence intervals) were calculated using the MOTE package in $R$ (Buchanan, Gillenwaters, Scofield, \& Valentine, 2019) with $t$-values acquired via Rubin's Rules across all imputed datasets (Rubin, 2004). Within- 
group effect size was calculated for likelihood of future NSSI $(d z)$, and the mean, median, standard deviation, minimum, and maximum values were reported within each condition for both frequency outcomes (NSSI and SI).

As pre-registered sensitivity analyses, we conducted these same analyses on our original, non-imputed dataset (i.e., excluded data from "missing" participants at post-intervention and follow-up time points) and within the subset of participants who completed an entire online program - either the intervention or the control $(N=452)$.

\section{Deviations from Pre-Registration}

While all conducted analyses aligned very closely with our pre-registered analytic plan, there were a few, minimal deviations. First, our final sample $(N=565)$ is 65 participants greater than our original, pre-registered trial size of 500. This deviation can primarily be attributed to a large group of qualifying participants completing our eligibility survey within a few hours of a study advertisement gaining unexpected traction on Instagram. As soon as we became aware that we had passed our pre-registered threshold, we stopped participant recruitment.

For our multiple imputation algorithms, we were unable to include one of the variables that we pre-registered (lifetime frequency of suicidal ideation) when creating imputed datasets for our past 3-month frequency of suicidal ideation outcome. Due to a handful of extremely high values in the baseline data (e.g. 1,000,000,000), the logical bounds for this variable were wide enough that the imputation algorithm continued to resample indefinitely (Honaker, King, \& Blackwell, 2011). As such, we were unable to include this variable when performing the final imputation process.

Finally, we considered a fifth, non-pre-registered data quality criteria when excluding participants: explicit participant self-disclosure of skipping through, or not reading, survey 
content (e.g., typing "i didn't read any of this" in an open-response section), which occurred in a handful of participants $(n=5)$. We did not explicitly ask participants whether or not they had read the survey and online programs (and, thus, did not pre-register this as a criterion for exclusion). However, in reading through open-ended responses, we noticed 5 participants spontaneously endorse this behavior; we excluded these 5 participants from the present analyses.

\section{Exploratory Analyses}

Exploratory analyses were conducted using two additional outcomes of direct clinical relevance: first, desire for future NSSI - measured immediately after completing an online program; second, self-hatred-measured immediately following the online program and again at 3 month follow-up. Similar procedures were followed for these two exploratory outcomes as with the likelihood of future NSSI outcome above. First, we checked assumptions for multiple regression (e.g., homoscedasticity), where study condition and baseline values were included as predictors for each respective outcome. If assumptions were met, we proceeded with interpretation of results. If assumptions were violated, we conducted additional analyses with robust standard errors using the sandwich package in R (Zeileis, Köll, \& Graham, 2020; Zeileis, 2004).

\section{Results}

\section{Sample Demographics}

Demographics for the final sample of $N=565$ included participants are reported in Table 1. When asked to identify their gender, race/ethnicity, participants were given the option to select multiple responses. As such, among this group of adolescents $\left(M_{\mathrm{age}}=14.95, S D_{\mathrm{age}}=0.98\right)$, $37.35 \%$ identified with a gender other than the one they were assigned at birth. The three most commonly endorsed genders were girl/woman (66.37\%), nonbinary $(19.29 \%)$, and "not sure" 
(10.62\%). The three most commonly endorsed sexual orientations were bisexual $(34.87 \%)$, homosexual (15.04\%), and heterosexual (11.86\%). Finally, the three most commonly endorsed races/ethnicities were White/Caucasian (75.04\%), Hispanic/Latinx (21.06\%), and Black/African American (9.73\%). Demographic information by condition is presented in Table 2.

[Table 1]

[Table 2]

\section{Dropout and Acceptability of Online Programs}

Of the 565 eligible adolescents who were randomized to an online program, 286 participants were randomized to SAVE and 279 participants were randomized to the control group. 452 completed an online program in-full $(80.00 \%)-232$ completed SAVE $(81.11 \%$ of those randomized) and 220 completed the control program ( $78.85 \%$ of those randomized). No significant differences were detected in dropout rates between the two groups, either for outcomes measured immediately following the online programs $(20.98 \%$ missing in SAVE, $21.51 \%$ missing in control, $p=.96$ ) or for follow-up outcomes measured three months later (58.04\% missing in SAVE, 61.29\% missing in control, $p=.48$ ). Participants in both conditions rated their respective online programs as acceptable, per pre-registered criteria (i.e., all item means of the Program Feedback Scale $\geq 3.5$; see Table 3 for full scale and item-level descriptives by condition).

[Table 3]

\section{Pre-Registered Analyses}

\section{Likelihood of Future NSSI (pre-registered, secondary outcome)}

All assumption checks for interpreting multiple regression were met (see Supplemental materials for details). After correcting for multiple tests, the change in self-reported likelihood of 
future NSSI from pre- to post-intervention did not differ between the SAVE and control groups $(d=-0.16,95 \%$ CI $-0.33,0.002, p=0.16)$. With respect to within-group effect sizes, selfreported likelihood of future NSSI significantly decreased from pre to post online program $\left(M_{\text {diff }}\right.$ $\left.=-0.63, \mathrm{SE}_{\text {diff }}=0.06\right)$ among SAVE participants $(d=-0.62,95 \% \mathrm{CI}-0.53,-0.29, p<.001)$. Similarly, likelihood of future NSSI significantly decreased from pre-program to post-program $\left(M_{\text {diff }}=-0.40, \mathrm{SE}_{\text {diff }}=0.06\right)$ among control group participants $(d=-0.41,95 \% \mathrm{CI}-0.75,-0.50, p$ $<.001)$.

\section{Past 3-Month Frequency NSSI (pre-registered, primary outcome)}

Prior to interpreting results, we calculated AIC and BIC values associated with the four possible model types for the NSSI frequency outcome (Poisson, negative binomial, zero-inflated Poisson, zero-inflated negative binomial). Across all 59 imputed datasets, AIC values were lowest for the zero-inflated negative binomial regression model. BIC values were lowest for the zero-inflated negative binomial regression model in 26 of the 59 imputed datasets, and lowest for the negative binomial regression model in the remaining 33 imputed datasets. Taking both AIC and BIC values into consideration, we interpreted the results of our zero-inflated negative binomial model. However, the zero-inflated negative binomial and the negative binomial models produced the same pattern of results for this outcome. Change in past 3-month frequency of NSSI from pre-intervention to 3-month follow-up did not significantly differ between SAVE and control-group participants $(d=-0.04,95 \%$ CI $-0.20,0.13, p=0.67)$. Means, median, standard deviation, and range of scores are reported by condition in Table 4.

[Table 4]

Past 3-Month Frequency Suicidal Ideation (pre-registered, secondary outcome) 
AIC values were lowest for the zero-inflated negative binomial regression in all 60 imputed datasets for the past 3-month frequency of suicidal ideation outcome variable. Similarly, BIC values were lowest for the zero-inflated negative binomial model among a majority (39) of the imputed datasets. As such, we interpreted the results of the zero-inflated negative binomial model. Change in past 3-month frequency of suicidal ideation from pre-intervention to 3-month follow-up did not significantly differ between SAVE and control-group participants $(d=0.11$, 95\% CI $-0.06,0.27, p=0.21)$. Means, median, standard deviation, and range of scores are reported by condition in Table 5 .

\section{[Table 5]}

\section{Sensitivity Analyses}

We ran these same, pre-registered analyses in the original, non-imputed dataset (i.e., dropping missing observations) to evaluate the robustness of our results. Consistent with our findings using the imputed data, change in self-reported likelihood of future NSSI from pre- to post-intervention did not significantly differ between conditions after correcting for multiple tests $(d=-0.17,95 \% \mathrm{CI}-0.34,-0.005, p=0.13)$, change in past 3 -month frequency of NSSI from pre-intervention to 3-month follow-up did not significantly differ between conditions $(d=0.07$, $95 \% \mathrm{CI}-0.09,0.24, p=0.38)$, and change in past 3-month frequency of suicidal ideation from pre-intervention to 3-month follow-up did not significantly differ between conditions $(d=0.09$, $95 \%$ CI $-0.08,0.25, p=0.29)$.

Additionally, we tested whether our pattern of results differed among adolescents who had completed an online program (i.e., a "completers" analysis, $N=452$ ). After correcting for multiple tests, there were no significant between-group differences for: change in likelihood of future NSSI from pre- to post-intervention $(d=-0.19,95 \% \mathrm{CI}-0.38,-0.006, p=0.13)$, change in 
past 3-month frequency of NSSI from pre-intervention to follow-up $(d=-0.01,95 \%$ CI -0.20 , $0.17, p=0.88$ ), or change in past 3-month frequency of suicidal ideation from pre-intervention to follow-up $(d=0.10,95 \%$ CI $-0.09,0.28, p=0.31)$. Thus, results for "completers" were consistent with full sample results.

\section{Exploratory Analyses}

Lastly, we evaluated effects of the SAVE intervention on two clinically relevant outcomes related to SITBs in adolescents-desire to stop future NSSI and self-hatred. For the first of these outcomes (desire to stop future NSSI), we verified assumptions for multiple regression were met prior to interpreting results. Relative to participants in the control group, SAVE participants reported significant increases in their desire to stop future NSSI from pre- to post-intervention $(d=0.25,95 \%$ CI $0.08,0.41, p=0.003)$. Within groups, both SAVE participants $(d=0.39,95 \%$ CI $0.27,0.51, p<.001)$ and control-group participants $(d=0.15,95 \%$ CI $0.03,0.27, p=0.013$ ) reported significant increases in their desire to stop future NSSI from pre- to post-intervention.

While evaluating effects of the SAVE intervention on self-hatred at post-intervention and follow-up, we discovered severe heteroscedasticity in our models for both time points. As such, we conducted additional analyses using robust standard errors (Zeileis, Köll, \& Graham, 2020; Zeileis, 2004). Relative to participants in the control group, SAVE participants reported significant decreases in self-hatred from pre- to post-intervention $(d=-0.35,95 \%$ CI $-0.51,-0.18$, $p<.001)$. However, this between-groups effect was no longer significant at 3-month follow-up ( $d$ $=0.009,95 \% \mathrm{CI}-0.16,0.17, p=0.91)$. Looking at change from pre-intervention to 3-month follow-up, we found significant decreases in self-hate in both the SAVE group $(d=-0.27,95 \%$ CI $-0.38,-0.15, p<.001)$ and the control group $(d=-0.23,95 \%$ CI $-0.35,-0.11, p<.001)$. 


\section{Discussion}

Recent research indicates that most existing treatments do not meaningfully reduce selfinjurious thoughts and behaviors (SITBs). Moreover, existing treatments are not accessed by most adolescents. To help overcome traditional barriers to treatment, this randomized-controlled trial tested whether a half-hour, self-guided, online intervention designed to reduce the frequency of self-injurious thoughts and behaviors ("Project SAVE") could reduce non-suicidal self-injury, suicidal thoughts, and self-hate in adolescents with a recent history of non-suicidal self-injury (NSSI) and negative self-beliefs. Project SAVE was rated as highly acceptable among participants, and $80 \%$ of those randomized to Project SAVE completed the program in fullstrongly suggesting SAVE's feasibility and acceptability as a fully self-guided, online support for adolescents engaging in NSSI. Compared to the attention-matched control intervention, Project SAVE predicted significantly greater desires to stop future NSSI at post-intervention $(d=$ $0.25)$, as well as significant reductions in self-hate $(d=-0.35)$ from pre-to-post-intervention. However, Project SAVE did not influence perceived likelihood of engaging in future NSSI compared to the control, and no differences emerged in 3-month SITBs by intervention condition.

Project SAVE's non-significant effects across all 3-month outcomes-including frequency of past-3-month NSSI and suicidal ideation - align with more than five decades of randomized controlled trials, which have largely failed to identify interventions that meaningfully reduce SITBs over time (Fox, Huang, et al., 2020). Indeed, intervention effects on SITBs are weaker for children and adolescents than for adults, and SITB treatments evaluated against active control conditions (versus no-treatment or waitlists) often produce null results, as observed here (Fox, Huang, et al., 2020). Moreover, all recruitment and follow-up data collection 
occurred at the height of the COVID-19 pandemic in the United States. Amid social isolation, economic recession, school closures, and other pandemic-related stressors, any intervention's capacity to reduce SITBs - often the only coping strategy that adolescents can reliably accessmight be limited.

Nonetheless, present results carry practical importance for several reasons. Many of this study's design features suggest high feasibility — and underscore the need — for large-scale, rigorous evaluations of novel SITB treatments for youth, including large sample sizes; preregistration of all study procedures and analysis plans; and sharing of study materials to improve reproducibility. The present trial $(N=565)$ utilizes a substantially larger sample size than a vast majority of SITB intervention trials conducted in youth to date (Brent et al., 2013; Glenn et al., 2015, 2019) - many of which enrolled fewer than 80 total participants. Thus, this project aligns with calls to increase sample sizes, thereby improving replicability, within clinical psychology research (Reardon et al., 2019). This trial also heeded many recommendations for conducting clinically-useful RCTs for SITB interventions, including calls to specify intervention effects on pre-registered, versus exploratory, outcome variables. Here, Project SAVE did not significantly reduce its pre-registered primary or secondary outcomes: past 3-month frequencies of NSSI or suicidal ideation. Further, all study materials, code, and de-identified data are available online (see https://osf.io/wfdzp/). Finally, the present trial represents one of relatively few existing trials designed to test SITB interventions for youth (only $17.45 \%$ of RCTs of SITB treatments have focused on children; Fox, Huang, et al., 2020).

Perhaps most importantly, Project SAVE was both highly acceptable and produced shortterm improvements in clinically-relevant domains, including self-hate and desire to stop selfharming in the future. Given the known volatility of SITBs, which often shift from day-to-day 
and even hour-to-hour, acceptable, low-intensity interventions that produce even short-term relief have clinical utility. Many youth have needs for interventions that can offer immediate support, and that may confer in-the-moment benefits - particularly those that they find to be acceptable, easy-to-use, and non-invasive. Per acceptability ratings, Project SAVE fits each of these criteria. Because the program also produced short-term increases in desires to stop selfharming, future research may seek to evaluate Project SAVE's potential to supplement other interventions that provide concrete strategies for disengaging with SITBs (e.g., safety planning) across a variety of treatment settings. For example, additional trials might compare treatment as usual plus SAVE, versus treatment as usual alone, in an emergency department (ED) care context. Such trials could also assess the cost-effectiveness of deploying SAVE in the ED; because SAVE requires no clinician, it may be offered to patients without placing additional staffing burdens on hospitals, positioning it as a cost-free, sustainable resource for EDsalthough present results suggest that additional supports may be necessary to sustain benefits over time. Even beyond emergency departments, the reality remains that most youth with SITBs access no treatment at all. As such, resources like SAVE—-which is anonymous, immediately accessible from any location, and acceptable to youth engaging in NSSI-may still offer in-themoment support to youth without access to other treatment. These possibilities suggest many avenues for research testing Project SAVE and similar interventions as free, low-intensity interventions for youth struggling with SITBs.

Moreover, because we obtained a waiver of parental consent from the University IRB, eligible adolescents were empowered to assent to participate in these interventions in the absence of parental consent. This decision parallels laws in many states in the U.S. which allow for adolescents aged 12 and above to pursue mental and physical healthcare in the absence of 
parent/guardian involvement. Research indicates that this choice may increase the likelihood that adolescents, and particularly minoritized adolescents, will pursue and be honest in mental health treatment (Samargia et al., 2006; Smith et al., 2021; Wilson \& Deane, 2012). Given that the SSIs tested here pose minimal risk to safety and may increase critical access to mental health support among adolescents, this choice represents a novel and important aspect of the present study. This, in combination with recruitment efforts designed to reach LGBTQ+ youth, culminated in a high number of participating LGBTQ+ and gender-diverse youth relative to the general population. Considering the numerous additional barriers to mental health treatments that many LGBTQ+ and gender-diverse youth face (Brown et al., 2016), increased representation of these groups is a significant strength of this intervention trial.

Several limitations of the present study warrant consideration. First, participation in these interventions required both Internet access and adolescent interest. Although most adolescents in the U.S. report access to the Internet, including via access to a desktop/laptop computer (88\%) and smartphone (95\%; Anderson \& Jiang, 2018), differences in Broadband (high-speed) access occur across socioeconomic status, education, U.S. region (e.g., lower access in rural regions, lower access on Tribal lands), and race/ethnicity — although there is some evidence that these disparities are decreasing (Bauerly et al., 2019). Although SSIs overcome many barriers to treatment, they do rely on Internet access and thus remain limited by differences in access to this resource. Second, $20 \%$ of randomized participants did not complete either intervention, and only $42 \%$ of participants completed the follow-up assessment three months after the intervention. Notably, an $80 \%$ completion rate for a fully self-guided, online intervention compares very favorably to most other digital mental health supports, which show engagement rates postdownload of 1-28\% (Fleming et al., 2018); this study's level of dropout also parallels other SITB 
treatments (Fox, Huang, et al., 2020). Additionally, we used multiple imputation techniques to provide a more conservative intent-to-treat approach than other common approaches to missing data (e.g. listwise deletion, last observation carried forward; Rubin, 2004); nonetheless, results may look different if including those who did not complete the follow-up assessment. Third, generalizability of the present results may be limited in some cases due to sample demographics. While LGBTQ+ and gender-diverse adolescents were well-represented within the present sample, only $5 \%$ of our participants were boys/men. The present sample's overrepresentation of specific groups (e.g., girls/women, non-binary adolescents), and the underrepresentation of others (e.g., boys/men), is consistent with higher rates of NSSI and suicidal ideation among girls/women, sexual minority, and gender minority adolescents (Bakken \& Gunter, 2012; Fox, Choukas-Bradley, et al., 2020). Lower representation of boys/men is also a common pattern among other studies offering digital mental health supports (Kramer et al., 2014; Sung et al., 2021; van der Zanden et al., 2012; Schleider et al., 2021); however, we cannot assume the present study's results are generalizable to adolescent boys. Additionally, the most commonly endorsed racial/ethnic identity within the present sample was White (75\%). Research examining differences in NSSI prevalence between racial and ethnic groups has found mixed results — with some research indicating higher prevalence within non-Hispanic White youth, relative to Hispanic and African American (Rojas-Velasquez et al., 2021), as well as Asian (Fox, ChoukasBradley, et al., 2020) peers. However, given pervasive racial and ethnic disparities in access to youth mental health treatments (Cummings \& Druss, 2011; Elster et al., 2003), single-session intervention research should specifically prioritize increasing access to mental health supports among youth of color. Fourth, two of our pre-registered outcome variables (past 3-month frequencies of NSSI and suicidal ideation) were based on retrospective self-report data. Some 
evidence suggests moderate to high reliability among similar retrospective, self-report itemsincluding number of days of lifetime suicidal ideation (ICCs = .51 - .74; Fox, Harris, et al., 2020; Nock et al., 2007) and number of days of past-year suicidal ideation (ICC $=.78$; Fox, Harris, et al., 2020; Nock et al., 2007). As both of our pre-registered outcomes were measured across a shorter time frame (i.e., past 3 months), it is likely the case that reliability is even higher among these variables. However, any retrospective, self-report data may be flawed. Lastly, as mentioned above, this study was conducted amid major, and for many, unprecedented life stressors across a range of dimensions; it remains unclear whether and the degree to which Project SAVE may have impacted our outcomes of interest outside of the context of COVID-19.

Two final, important considerations for interpreting the present results include: statistical power and the use of an active control condition. First, true effect sizes for the Project SAVE single-session intervention on NSSI and suicidal ideation could be smaller than anticipated at this trial's outset. One meta-analysis of randomized trials evaluating single-session interventions found a small-to-medium effect across a broad range of youth mental health problems (Hedges's $g=.32$; Schleider \& Weisz, 2017); a recent, larger-scale randomized trial $(N=2,452)$ of two web-based, single-session intervention programs indicate significant, small long-term improvements in depression symptoms and hopelessness 3 months later, relative to an active control $(d \mathrm{~s}=.18, p \mathrm{~s}<.001 ;$ Schleider, et al., 2021). Thus, future trials of digital single-session intervention programs should be properly powered to detect effect sizes within this range-likely approaching 1,000 participants or more. Second, for ethical reasons, the present trial compared the Project SAVE intervention to an active, supportive therapy control program rather than using a waiting list control. Relative to receiving no treatment - as is the case for many youth who experience self-injurious thoughts and behaviors - it is possible that a single session of 
supportive therapy provided non-zero benefit to the present control group participants. One large- $\mathrm{N}$ trial that utilized an identical online, supportive therapy control program showed significant within-group reductions in depression over 3 months (within-group $d \mathbf{s}=-.35$; Schleider et al., 2021). However, any potential benefits introduced by the active control program are indistinguishable from the possible effects of time.

Despite these limitations and considerations, this study represents the first attempt to create and test a youth-initiated, web-based SSI to reduce SITBs among a diverse sample of adolescents. This approach is particularly important in light of major barriers to traditional treatment access among adolescents and in light of poor treatment efficacy across traditional approaches. Results highlight that SSIs, and particularly Project SAVE, is an acceptable resource for adolescents engaging in SITBs — with short-term effects on clinically-relevant outcomes including self-hate. Future research may seek to build on SAVE to help promote stronger longerterm outcomes. 


\section{References}

Anderson, M., \& Jiang, J. (2018). Teens, social media \& technology 2018. Pew Research Center, 31(2018), 1673-1689.

Asarnow, J. R., Porta, G., Spirito, A., Emslie, G., Clarke, G., Wagner, K. D., Vitiello, B., Keller, M., Birmaher, B., McCracken, J., Mayes, T., Berk, M., \& Brent, D. A. (2011). Suicide attempts and nonsuicidal self-injury in the treatment of resistant depression in adolescents: Findings from the TORDIA study. Journal of the American Academy of Child and Adolescent Psychiatry, 50(8), 772-781.

Bakken, N. W., \& Gunter, W. D. (2012). Self-cutting and suicidal ideation among adolescents: Gender differences in the causes and correlates of self-injury. Deviant Behavior, 33(5), $339-356$.

Batejan, K. L., Jarvi, S. M., \& Swenson, L. P. (2015). Sexual orientation and non-suicidal selfinjury: a meta-analytic review. Archives of Suicide Research: Official Journal of the International Academy for Suicide Research, 19(2), 131-150.

Bauerly, B. C., McCord, R. F., Hulkower, R., \& Pepin, D. (2019). Broadband access as a public health issue: The role of law in expanding broadband access and connecting underserved communities for better health outcomes. Journal of Law, Medicine \& Ethics, 47(S2), 39-42. https://doi.org/10.1177/1073110519857314

Benjamini, Y., \& Hochberg, Y. (1995). Controlling the false discovery rate: A practical and powerful approach to multiple testing. Journal of the Royal statistical society: series $B$ (Methodological), 57(1), 289-300.

Brausch, A. M., \& Girresch, S. K. (2012). A Review of Empirical Treatment Studies for Adolescent Nonsuicidal Self-Injury. In Journal of Cognitive Psychotherapy (Vol. 26, Issue 
1, pp. 3-18). https://doi.org/10.1891/0889-8391.26.1.3

Brent, D. A., Greenhill, L. L., Compton, S., Emslie, G., Wells, K., Walkup, J. T., Vitiello, B., Bukstein, O., Stanley, B., Posner, K., Kennard, B. D., Cwik, M. F., Wagner, A., Coffey, B., March, J. S., Riddle, M., Goldstein, T., Curry, J., Barnett, S., ... Blake Turner, J. (2009). The Treatment of Adolescent Suicide Attempters Study (TASA): Predictors of suicidal events in an open treatment trial. Journal of the American Academy of Child \& Adolescent Psychiatry, 48(10), 987-996. https://doi.org/10.1097/chi.0b013e3181b5dbe4

Brent, D. A., McMakin, D. L., Kennard, B. D., Goldstein, T. R., Mayes, T. L., \& Douaihy, A. B. (2013). Protecting adolescents from self-harm: a critical review of intervention studies. Journal of the American Academy of Child and Adolescent Psychiatry, 52(12), 1260-1271.

Brown, A., Rice, S. M., Rickwood, D. J., \& Parker, A. G. (2016). Systematic review of barriers and facilitators to accessing and engaging with mental health care among at-risk young people. Asia-Pacific Psychiatry: Official Journal of the Pacific Rim College of Psychiatrists, 8(1), 3-22.

Buchanan E, Gillenwaters A, Scofield J, Valentine K (2019). MOTE: Measure of the Effect: Package to assist in effect size calculations and their confidence intervals. $\mathrm{R}$ package version 1.0.2, https://cran.r-project.org/web/packages/MOTE/index.html.

Burke, T. A., Fox, K., Kautz, M. M., Rodriguez-Seijas, C., Bettis, A. H., \& Alloy, L. B. (2021). Self-critical and self-punishment cognitions differentiate those with and without a history of nonsuicidal self-injury: An ecological momentary assessment study. Behavior Therapy, $52(3), 686-697$.

Cox, L. J., Stanley, B. H., Melhem, N. M., Oquendo, M. A., Birmaher, B., Burke, A., Kolko, D. J., Zelazny, J. M., Mann, J. J., Porta, G., \& Brent, D. A. (2012). A longitudinal study of 
nonsuicidal self-injury in offspring at high risk for mood disorder. The Journal of Clinical Psychiatry, 73(6), 821-828.

Cummings, J. R., \& Druss, B. G. (2011). Racial/ethnic differences in mental health service use among adolescents with major depression. Journal of the American Academy of Child and Adolescent Psychiatry, 50(2), 160-170.

Dobias, M.L., Fox, K.R., \& Schleider, J.L. (2020). Project SAVE: A single-session intervention targeting self-injurious behavior in adolescents. doi: 10.17605/OSF.IO/WFDZP.

Doyle, L., Sheridan, A., \& Treacy, M. P. (2017). Motivations for adolescent self-harm and the implications for mental health nurses. Journal of Psychiatric and Mental Health Nursing, 24(2-3), 134-142.

Elster, A., Jarosik, J., VanGeest, J., \& Fleming, M. (2003). Racial and ethnic disparities in health care for adolescents: a systematic review of the literature. Archives of Pediatrics \& Adolescent Medicine, 157(9), 867-874.

Esposito-Smythers, C., Spirito, A., Kahler, C. W., Hunt, J., \& Monti, P. (2011). Treatment of cooccurring substance abuse and suicidality among adolescents: A randomized trial. Journal of Consulting and Clinical Psychology, 79(6), 728-739.

Fleming, T., Bavin, L., Lucassen, M., Stasiak, K., Hopkins, S., \& Merry, S. (2018). Beyond the trial: Systematic review of real-world uptake and engagement with digital self-help interventions for depression, low mood, or anxiety. Journal of Medical Internet Research, 20(6), e199.

Fox, K. R., Choukas-Bradley, S., Salk, R. H., Marshal, M. P., \& Thoma, B. C. (2020). Mental health among sexual and gender minority adolescents: Examining interactions with race and ethnicity. Journal of Consulting and Clinical Psychology, 88(5), 402-415. 
Fox, K. R., Harris, J. A., Wang, S. B., Millner, A. J., Deming, C. A., \& Nock, M. K. (2020). Self-Injurious Thoughts and Behaviors Interview-Revised: Development, reliability, and validity. Psychological Assessment, 32(7), 677-689.

Fox, K. R., Huang, X., Guzmán, E. M., Funsch, K. M., Cha, C. B., Ribeiro, J. D., \& Franklin, J. C. (2020). Interventions for suicide and self-injury: A meta-analysis of randomized controlled trials across nearly 50 years of research. Psychological Bulletin, 146(12), 11171145.

Fox, K. R., Ribeiro, J. D., Kleiman, E. M., Hooley, J. M., Nock, M. K., \& Franklin, J. C. (2018). Affect toward the self and self-injury stimuli as potential risk factors for nonsuicidal selfinjury. Psychiatry Research, 260, 279-285.

Franklin, J. C., Huang, X., Fox, K. R., \& Ribeiro, J. D. (2018). What suicide interventions should target. Current Opinion in Psychology, 22, 50-53.

Glenn, C. R., Esposito, E. C., Porter, A. C., \& Robinson, D. J. (2019). Evidence base update of psychosocial treatments for self-injurious thoughts and behaviors in youth. Journal of Clinical Child and Adolescent Psychology, 48(3), 357-392.

Glenn, C. R., Franklin, J. C., \& Nock, M. K. (2015). Evidence-based psychosocial treatments for self-injurious thoughts and behaviors in youth. Journal of Clinical Child and Adolescent Psychology, 44(1), 1-29.

Gonzales, A. H., \& Bergstrom, L. (2013). Adolescent non-suicidal self-injury (NSSI) interventions. In Journal of Child and Adolescent Psychiatric Nursing, 26(2), 124-130. https://doi.org/10.1111/jcap.12035

Granboulan, V., Roudot-Thoraval, F., Lemerle, S., \& Alvin, P. (2001). Predictive factors of postdischarge follow-up care among adolescent suicide attempters. Acta Psychiatrica 
Scandinavica, 104(1), 31-36.

Halamová, J., S., Kanovský, M., Jurková, V., Kupeli, N. (2018). Effect of a short-term online version of a mindfulness-based intervention on self-criticism and self-compassion in a nonclinical sample. Studia Psychologica, 60(4), 259-273.

https://doi.org/10.21909/sp.2018.04.766

Honaker J, King G, Blackwell M (2011). Amelia II: A program for missing data. Journal of Statistical Software, 45(7), 1-47. http://www.jstatsoft.org/v45/i07/.

Hooley, J. M., Fox, K. R., Wang, S. B., \& Kwashie, A. N. D. (2018). Novel online daily diary interventions for nonsuicidal self-injury: a randomized controlled trial. BMC Psychiatry, $18(1), 264$.

Husky, M. M., Olfson, M., He, J.-P., Nock, M. K., Swanson, S. A., \& Merikangas, K. R. (2012). Twelve-month suicidal symptoms and use of services among adolescents: Results from the National Comorbidity Survey. Psychiatric Services, 63(10), 989-996.

Kidd, S. A., Howison, M., Pilling, M., Ross, L. E., \& McKenzie, K. (2016). Severe Mental Illness in LGBT Populations: A Scoping Review. Psychiatric Services , 67(7), 779-783.

Klonsky, E. D. (2007). The functions of deliberate self-injury: a review of the evidence. Clinical Psychology Review, 27(2), 226-239.

Kovacs, M. (2015). Children's depression inventory (CDI and CDI 2). In The Encyclopedia of Clinical Psychology (pp. 1-5). John Wiley \& Sons, Inc. https://doi.org/10.1002/9781118625392.wbecp419

Kramer, J., Conijn, B., Oijevaar, P., \& Riper, H. (2014). Effectiveness of a web-based solutionfocused brief chat treatment for depressed adolescents and young adults: Randomized controlled trial. Journal of Medical Internet Research, 16(5), e141. 
Labelle, R., Pouliot, L., \& Janelle, A. (2015). A systematic review and meta-analysis of cognitive behavioural treatments for suicidal and self-harm behaviours in adolescents. Canadian Psychology/Psychologie Canadienne, 56(4), 368-378.

Laye-Gindhu, A., \& Schonert-Reichl, K. A. (2005). Nonsuicidal self-harm among community adolescents: Understanding the "whats" and "whys" of self-harm. In Journal of Youth and Adolescence, 34(5), 447-457. https://doi.org/10.1007/s10964-005-7262-z

Lizardi, D., \& Stanley, B. (2010). Treatment engagement: a neglected aspect in the psychiatric care of suicidal patients. Psychiatric Services, 61(12), 1183-1191.

Luoma, J. B., \& Platt, M. G. (2015). Shame, self-criticism, self-stigma, and compassion in Acceptance and Commitment Therapy. In Current Opinion in Psychology, 2, 97-101. https://doi.org/10.1016/j.copsyc.2014.12.016

Marshal, M. P., Dietz, L. J., Friedman, M. S., Stall, R., Smith, H. A., McGinley, J., Thoma, B. C., Murray, P. J., D’Augelli, A. R., \& Brent, D. A. (2011). Suicidality and depression disparities between sexual minority and heterosexual youth: a meta-analytic review. The Journal of Adolescent Health, 49(2), 115-123.

McCabe, R., Garside, R., Backhouse, A., \& Xanthopoulou, P. (2018). Effectiveness of brief psychological interventions for suicidal presentations: a systematic review. $B M C$ Psychiatry, 18(1), 120.

Nock, M. K. (2010). Self-injury. Annual Review of Clinical Psychology, 6, 339-363.

Nock, M. K., Borges, G., Bromet, E. J., Cha, C. B., Kessler, R. C., \& Lee, S. (2008). Suicide and suicidal behavior. Epidemiologic Reviews, 30, 133-154.

Nock, M. K., Green, J. G., Hwang, I., McLaughlin, K. A., Sampson, N. A., Zaslavsky, A. M., \& Kessler, R. C. (2013). Prevalence, correlates, and treatment of lifetime suicidal behavior 
among adolescents: results from the National Comorbidity Survey Replication Adolescent Supplement. JAMA Psychiatry, 70(3), 300-310.

Nock, M. K., Holmberg, E. B., Photos, V. I., \& Michel, B. D. (2007). Self-Injurious Thoughts and Behaviors Interview: development, reliability, and validity in an adolescent sample. Psychological Assessment, 19(3), 309-317.

Ougrin, D., Tranah, T., Stahl, D., Moran, P., \& Asarnow, J. R. (2015). Therapeutic interventions for suicide attempts and self-harm in adolescents: Systematic review and meta-analysis. Journal of the American Academy of Child and Adolescent Psychiatry, 54(2), 97-107.e2.

Pfeiffer, P. N., Brandfon, S., Garcia, E., Duffy, S., Ganoczy, D., Myra Kim, H., \& Valenstein, M. (2014). Predictors of suicidal ideation among depressed Veterans and the interpersonal theory of suicide. Journal of Affective Disorders, 152-154, 277-281.

Piacentini, J., Rotheram-Borus, M. J., Gillis, J. R., Graae, F., Trautman, P., Cantwell, C., GarciaLeeds, C., \& Shaffer, D. (1995). Demographic predictors of treatment attendance among adolescent suicide attempters. Journal of Consulting and Clinical Psychology, 63(3), 469473.

Reardon, K. W., Smack, A. J., Herzhoff, K., \& Tackett, J. L. (2019). An N-pact factor for clinical psychological research. Journal of Abnormal Psychology, 128(6), 493-499.

Ribeiro, J. D., Franklin, J. C., Fox, K. R., Bentley, K. H., Kleiman, E. M., Chang, B. P., \& Nock, M. K. (2016). Self-injurious thoughts and behaviors as risk factors for future suicide ideation, attempts, and death: a meta-analysis of longitudinal studies. Psychological Medicine, 46(2), 225-236. https://doi.org/10.1017/s0033291715001804

Rojas-Velasquez, D. A., Pluhar, E. I., Burns, P. A., \& Burton, E. T. (2021). Nonsuicidal selfinjury among African American and Hispanic adolescents and young adults: A systematic 
review. Prevention Science, 22(3), 367-377.

Rossouw, T. I., \& Fonagy, P. (2012). Mentalization-based treatment for self-harm in adolescents: a randomized controlled trial. Journal of the American Academy of Child and Adolescent Psychiatry, 51(12), 1304-1313.e3.

Rubin, D. B. Multiple Imputation for Nonresponse in Surveys. (John Wiley \& Sons, 2004).

Samargia, L. A., Saewyc, E. M., \& Elliott, B. A. (2006). Foregone mental health care and selfreported access barriers among adolescents. The Journal of School Nursing: The Official Publication of the National Association of School Nurses, 22(1), 17-24.

Schleider, J. L., Dobias, M. L., Sung, J. Y., \& Mullarkey, M. C. (2020). Future directions in single-session youth mental health interventions. Journal of Clinical Child and Adolescent Psychology, 49(2), 264-278.

Schleider, J. L., Dobias, M., Sung, J., Mumper, E., \& Mullarkey, M. C. (2020). Acceptability and Utility of an Open-Access, Online Single-Session Intervention Platform for Adolescent Mental Health. JMIR Mental Health, 7(6), e20513.

Schleider, J. L., Mullarkey, M. C., Fox, K., Dobias, M., Shroff, A., Hart, E., \& Roulston, C. A. (2021). Single-session interventions for adolescent depression in the context of COVID-19: A nationwide randomized-controlled trial. https://doi.org/10.31234/osf.io/ved4p

Schleider, J. L., Mullarkey, M. C., \& Weisz, J. R. (2019). Virtual reality and web-based growth mindset interventions for adolescent depression: Protocol for a three-arm randomized trial. JMIR Research Protocols, 8(7), e13368.

Schleider, J. L., \& Weisz, J. R. (2017). Little treatments, promising effects? Meta-analysis of single-session interventions for youth psychiatric problems. Journal of the American Academy of Child and Adolescent Psychiatry, 56(2), 107-115. 
Schleider, J., \& Weisz, J. (2018). A single-session growth mindset intervention for adolescent anxiety and depression: 9-month outcomes of a randomized trial. Journal of Child Psychology and Psychiatry, and Allied Disciplines, 59(2), 160-170.

Schleider, J. L., \& Weisz, J. R. (2019). Sharing Feelings Project. Open Science Framework. https://doi.org/10.17605/OSF.IO/SWV5B

Signorell et al. (2020). DescTools: Tools for descriptive statistics. R package version 0.99.34, https://cran.r-project.org/package=DescTools.

Smith, D. M., Wang, S. B., Carter, M. L., Fox, K. R., \& Hooley, J. M. (2020). Longitudinal predictors of self-injurious thoughts and behaviors in sexual and gender minority adolescents. Journal of Abnormal Psychology, 129(1), 114-121.

Smith, D. M. Y., Lipson, S. M., Wang, S. B., \& Fox, K. R. (2021). Online Methods in Adolescent Self-Injury Research: Challenges and Recommendations. Journal of Clinical Child and Adolescent Psychology, 1-12.

Stanley, B., \& Brown, G. K. (2012). Safety planning intervention: A brief intervention to mitigate suicide risk. Cognitive and Behavioral Practice, 19(2), 256-264. https://doi.org/10.1016/j.cbpra.2011.01.001

Stewart, K. L., Darling, E. V., Yen, S., Stanley, B., Brown, G. K., \& Weinstock, L. M. (2019). Dissemination of the Safety Planning Intervention (SPI) to University Counseling Center Clinicians to Reduce Suicide Risk Among College Students. In Archives of Suicide Research (pp. 1-11). https://doi.org/10.1080/13811118.2018.1531797

Stiffman, A. R., Pescosolido, B., \& Cabassa, L. J. (2004). Building a Model to Understand Youth Service Access: The Gateway Provider Model. In Mental Health Services Research (Vol. 6, Issue 4, pp. 189-198). https://doi.org/10.1023/b:mhsr.0000044745.09952.33 
Sung, J. Y., Mumper, E., \& Schleider, J. L. (2021). Empowering Anxious Parents to Manage Child Avoidance Behaviors: Randomized Control Trial of a Single-Session Intervention for Parental Accommodation. JMIR Mental Health, 8(7), e29538.

Swannell, S. V., Martin, G. E., Page, A., Hasking, P., \& St John, N. J. (2014). Prevalence of nonsuicidal self-injury in nonclinical samples: systematic review, meta-analysis and metaregression. Suicide \& Life-Threatening Behavior, 44(3), 273-303.

Taylor, L. M. W., Oldershaw, A., Richards, C., Davidson, K., Schmidt, U., \& Simic, M. (2011). Development and pilot evaluation of a manualized cognitive-behavioural treatment package for adolescent self-harm. Behavioural and Cognitive Psychotherapy, 39(5), 619-625.

Trautman, P. D., Stewart, N., \& Morishima, A. (1993). Are adolescent suicide attempters noncompliant with outpatient care? Journal of the American Academy of Child and Adolescent Psychiatry, 32(1), 89-94.

Turnell, A. I., Fassnacht, D. B., Batterham, P. J., Calear, A. L., \& Kyrios, M. (2019). The SelfHate Scale: Development and validation of a brief measure and its relationship to suicidal ideation. Journal of Affective Disorders, 245, 779-787.

van der Zanden, R., Kramer, J., Gerrits, R., \& Cuijpers, P. (2012). Effectiveness of an online group course for depression in adolescents and young adults: a randomized trial. Journal of Medical Internet Research, 14(3), e86.

Whitlock, J., Muehlenkamp, J., Eckenrode, J., Purington, A., Baral Abrams, G., Barreira, P., \& Kress, V. (2013). Nonsuicidal self-injury as a gateway to suicide in young adults. The Journal of Adolescent Health, 52(4), 486-492.

Wilson, C. J., \& Deane, F. P. (2012). Brief report: Need for autonomy and other perceived barriers relating to adolescents' intentions to seek professional mental health care. Journal 
of Adolescence, 35(1), 233-237.

Zeileis A (2004). Econometric computing with HC and HAC covariance matrix estimators. Journal of Statistical Software, 11(10), 1-17. doi: 10.18637/jss.v011.i10.

Zeileis A, Köll S, Graham N (2020). Various versatile variances: An object-oriented implementation of clustered covariances in R. Journal of Statistical Software, 95(1), 1-36. doi: 10.18637/jss.v095.i01.

Zelkowitz, R. L., \& Cole, D. A. (2019). Self-criticism as a transdiagnostic process in nonsuicidal self-injury and disordered eating: Systematic review and meta-analysis. Suicide \& LifeThreatening Behavior, 49(1), 310-327. 
Table 1

Sample Demographics $(N=565)$

\begin{tabular}{|c|c|}
\hline & $\begin{array}{c}\text { Total Sample } \\
\text { M (SD) or N }(\%)\end{array}$ \\
\hline $\mathrm{N}$ & 565 \\
\hline Age & $14.95(0.98)$ \\
\hline \multicolumn{2}{|l|}{ Race/Ethnicity } \\
\hline American Indian or Alaska Native & $31(5.49 \%)$ \\
\hline Asian (including Asian Desi) & $41(7.26 \%)$ \\
\hline Black/African American & $55(9.73 \%)$ \\
\hline Hispanic/Latinx & $119(21.06 \%)$ \\
\hline Native Hawaiian or other Pacific Islander & $9(1.59 \%)$ \\
\hline White/Caucasian & $424(75.04 \%)$ \\
\hline Prefer not to answer & $5(0.88 \%)$ \\
\hline Other/Not Listed & $12(2.12 \%)$ \\
\hline \multicolumn{2}{|l|}{ Gender Identity Differs From Sex } \\
\hline Yes & $211(37.35 \%)$ \\
\hline No & $345(61.06 \%)$ \\
\hline Missing & $9(1.59 \%)$ \\
\hline \multicolumn{2}{|l|}{ Gender Identity } \\
\hline Female & $375(66.37 \%)$ \\
\hline Male & $49(8.67 \%)$ \\
\hline Transgender & $45(7.96 \%)$ \\
\hline Female to male transgender (FTM) & $34(6.01 \%)$ \\
\hline Trans male & $31(5.49 \%)$ \\
\hline Trans masculine & $31(5.49 \%)$ \\
\hline Trans feminine & $2(0.35 \%)$ \\
\hline Genderqueer & $33(5.84 \%)$ \\
\hline Gender expansive & $13(2.30 \%)$ \\
\hline Intersex & $2(0.35 \%)$ \\
\hline
\end{tabular}


Androgynous

Nonbinary

Two-spirited

Third gender

Agender

Not sure

Other/Not Listed

Missing

Sexual Orientation

Heterosexual

Homosexual

Bisexual

Pansexual

Queer

Asexual

Other/Not Listed

Unsure/Questioning

Does not use a label

Prefer not to answer

$$
\begin{gathered}
39(6.90 \%) \\
109(19.29 \%) \\
6(1.06 \%) \\
4(0.71 \%) \\
23(4.07 \%) \\
60(10.62 \%) \\
26(4.60 \%) \\
14(2.48 \%) \\
67(11.86 \%) \\
85(15.04 \%) \\
197(34.87 \%) \\
57(10.09 \%) \\
31(5.49 \%) \\
21(3.72 \%) \\
27(4.78 \%) \\
50(8.85 \%) \\
27(4.78 \%) \\
3(0.53 \%)
\end{gathered}
$$

Note. Gender and race/ethnicity categories were not mutually exclusive. 
Table 2

Sample Demographics by Condition

\begin{tabular}{|c|c|c|}
\hline \multirow{3}{*}{ Demographics } & \multicolumn{2}{|c|}{ Condition } \\
\hline & Placebo Control, $\mathrm{N}=279$ & Project SAVE, $N=286$ \\
\hline & $\mathrm{M}(\mathrm{SD})$ or $\mathrm{N}(\%)$ & $\mathrm{M}(\mathrm{SD})$ or $\mathrm{N}(\%)$ \\
\hline Age & $14.97(0.99)$ & $14.92(0.98)$ \\
\hline \multicolumn{3}{|l|}{ Race/Ethnicity } \\
\hline American Indian or Alaska Native & $19(6.81 \%)$ & $12(4.20 \%)$ \\
\hline Asian (including Asian Desi) & $25(8.96 \%)$ & $16(5.59 \%)$ \\
\hline Black/African-American & $30(10.75 \%)$ & $25(8.74 \%)$ \\
\hline Hispanic/Latinx & $60(21.50 \%)$ & $59(20.63 \%)$ \\
\hline Native Hawaiian or other Pacific Islander & $6(2.15 \%)$ & $3(1.05 \%)$ \\
\hline White/Caucasian & $204(73.12 \%)$ & $220(76.92 \%)$ \\
\hline Prefer not to answer & $4(1.43 \%)$ & $1(0.35 \%)$ \\
\hline Other/Not Listed & $8(2.87 \%)$ & $4(1.40 \%)$ \\
\hline \multicolumn{3}{|l|}{ Gender Identity Differs From Sex } \\
\hline Yes & $101(36.20 \%)$ & $110(38.46 \%)$ \\
\hline No & $172(61.65 \%)$ & $173(60.49 \%)$ \\
\hline Missing & $6(2.15 \%)$ & $3(1.05 \%)$ \\
\hline \multicolumn{3}{|l|}{ Gender Identity } \\
\hline Female & $186(66.67 \%)$ & $189(66.08 \%)$ \\
\hline Male & $25(8.96 \%)$ & $24(8.39 \%)$ \\
\hline Transgender & $23(8.24 \%)$ & $22(7.69 \%)$ \\
\hline Female to male transgender (FTM) & $18(6.45 \%)$ & $16(5.59 \%)$ \\
\hline Trans male & $15(5.38 \%)$ & $16(5.59 \%)$ \\
\hline
\end{tabular}




\section{Condition}

\begin{tabular}{|c|c|c|}
\hline Demographics & $\begin{array}{c}\text { Placebo Control, } \mathrm{N}=279 \\
\mathrm{M}(\mathrm{SD}) \text { or } \mathrm{N}(\%)\end{array}$ & $\begin{array}{c}\text { Project SAVE, } \mathrm{N}=286 \\
\mathrm{M}(\mathrm{SD}) \text { or } \mathrm{N}(\%)\end{array}$ \\
\hline Trans masculine & $18(6.45 \%)$ & $13(4.55 \%)$ \\
\hline Trans feminine & $1(0.36 \%)$ & $1(0.35 \%)$ \\
\hline Genderqueer & $19(6.81 \%)$ & $14(4.90 \%)$ \\
\hline Gender expansive & $7(2.51 \%)$ & $6(2.10 \%)$ \\
\hline Intersex & $1(0.36 \%)$ & $1(0.35 \%)$ \\
\hline Androgynous & $20(7.17 \%)$ & $19(6.64 \%)$ \\
\hline Nonbinary & $48(17.20 \%)$ & $61(21.33 \%)$ \\
\hline Two-spirited & $2(0.72 \%)$ & $4(1.40 \%)$ \\
\hline Third gender & $1(0.36 \%)$ & $3(1.05 \%)$ \\
\hline Agender & $10(3.58 \%)$ & $13(4.55 \%)$ \\
\hline Not sure & $26(9.32 \%)$ & $34(11.89 \%)$ \\
\hline Other/Not Listed & $9(3.23 \%)$ & $17(5.94 \%)$ \\
\hline Missing & $8(2.87 \%)$ & $6(2.10 \%)$ \\
\hline \multicolumn{3}{|l|}{ Sexual Orientation } \\
\hline Heterosexual & $38(13.62 \%)$ & $29(10.14 \%)$ \\
\hline Homosexual & $39(13.98 \%)$ & $46(16.08 \%)$ \\
\hline Bisexual & $107(38.35 \%)$ & $90(31.47 \%)$ \\
\hline Pansexual & $26(9.32 \%)$ & $31(10.84 \%)$ \\
\hline Queer & $10(3.58 \%)$ & $21(7.34 \%)$ \\
\hline Asexual & $7(2.51 \%)$ & $14(4.90 \%)$ \\
\hline Other/Not Listed & $15(5.38 \%)$ & $12(4.20 \%)$ \\
\hline Unsure/Questioning & $20(7.17 \%)$ & $30(10.49 \%)$ \\
\hline
\end{tabular}




\section{Condition}

\begin{tabular}{lcc}
\hline Demographics & $\begin{array}{c}\text { Placebo Control, N }=279 \\
\text { M (SD) or N (\%) }\end{array}$ & $\begin{array}{c}\text { Project SAVE, N }=286 \\
\text { M (SD) or N (\%) }\end{array}$ \\
\hline Does not use a label & $14(5.02 \%)$ & $13(4.55 \%)$ \\
Prefer not to answer & $3(1.08 \%)$ & $0(0.00 \%)$ \\
\hline
\end{tabular}

Note. Gender and race/ethnicity categories were not mutually exclusive. 
Table 3

Means and standard deviations of Program Feedback Scale items by condition

\begin{tabular}{lccc}
\hline & $\begin{array}{c}\text { Project SAVE } \\
\text { M (SD) }\end{array}$ & $\begin{array}{c}\text { Control Program } \\
\text { M (SD) }\end{array}$ & $\begin{array}{c}\text { Full Sample } \\
\text { M (SD) }\end{array}$ \\
\hline PFS item & $3.76(0.88)$ & $3.52(0.80)$ & $3.64(0.85)$ \\
Enjoy & $4.40(0.66)$ & $4.46(0.63)$ & $4.42(0.64)$ \\
Understood & $4.37(0.70)$ & $4.42(0.68)$ & $4.40(0.69)$ \\
Easy to use & $4.43(0.79)$ & $4.31(0.75)$ & $4.37(0.77)$ \\
Tried hardest & $4.15(0.96)$ & $3.97(0.94)$ & $4.06(0.95)$ \\
Helpful & $3.83(1.11)$ & $3.66(1.07)$ & $3.75(1.09)$ \\
Recommend to friend & $4.51(0.69)$ & $4.37(0.73)$ & $4.44(0.71)$ \\
Agree with message & $4.21(0.58)$ & $4.10(0.54)$ & $4.16(0.56)$ \\
PFS full scale & &
\end{tabular}

Note. Program Feedback Scale $=$ PFS. 
Table 4

Descriptives for past 3-month frequency of engaging in NSSI (total self-reported count) at baseline and follow-up by condition

\begin{tabular}{lccc}
\hline & Mean (SD) & Median & Range \\
\hline Baseline & & & \\
Project SAVE & $15.81(16.98)$ & 9.00 & $1.00-61.81$ \\
Control Program & $13.93(15.36)$ & 7.00 & $1.00-61.81$ \\
Follow-Up & & & \\
Project SAVE & $22.72(21.87)$ & 15.31 & $0.00-74.68$ \\
Control Program & $23.85(22.10)$ & 17.36 & $0.00-74.68$ \\
\hline
\end{tabular}

Note. Baseline medians are integer values because this outcome is a count variable. Follow-up medians are not integer values because of Amelia-II imputed values in place of missing data. Maximum values are often equivalent between conditions due to winsorizing of outliers. There are no significant differences between groups. 
Table 5

Descriptives for past 3-month frequency of experiencing suicidal ideation (total self-reported count) at baseline and follow-up by condition

\begin{tabular}{llcc}
\hline & Mean (SD) & Median & Range \\
\hline Baseline & & & \\
Project SAVE & $24.74(28.78)$ & 10.00 & $0.00-98.83$ \\
Control Program & $24.00(28.94)$ & 10.00 & $0.00-98.83$ \\
Follow-Up & & & \\
Project SAVE & $35.73(29.14)$ & 29.64 & $0.00-95.37$ \\
Control Program & $31.54(26.95)$ & 25.05 & $0.00-95.37$ \\
\hline
\end{tabular}

Note. Baseline medians are integer values because this outcome is a count variable. Follow-up medians are not integer values because of Amelia-II imputed values in place of missing data. Maximum values are often equivalent between conditions due to winsorizing of outliers. There are no significant differences between groups. 